\title{
LLLT Part 3: Critically Assessing Recent Published LLLT Device Trials: Limitations, Recommendations, and Conclusions
}

\author{
Sharon A. Keene, MD, FISHRS Tucson, Arizona, USA skeene@ishrs.org
}

In this final installment evaluating the science and application of LLLT to treat hair loss, evidence will be evaluated in the context of recent published data, recognized methods to measure treatment response, and known properties of biostimulation. Limitations of methodologies will be discussed, as well as how this information may be used to assist patients in decision making if they are considering the purchase of a cleared and legally distributed LLLT device.

Clinical trial data from randomized, controlled studies is the sine qua non of evidence used to determine effectiveness for any medical therapy. ${ }^{1}$ The U.S. FDA has established acceptable methods of assessing effective hair growth therapy when approving topical minoxidil, and similar methods for finasteride. These include before and after phototrichograms, and recommended time blinded global photo assessments. ${ }^{2}$ However, because LLLT devices go through a $510(\mathrm{k})$ clearance process, rather than pre-market or FDA approval, many of the LLLT devices that have been cleared and currently marketed in the United States have no clinical proof of efficacy. ${ }^{3}$ As licensed medical practitioners, doctors have an obligation and patients have an expectation that recommended medical treatments are, above all, safe, but also proven effective. Ethical considerations, as promoted by organized medicine, dictate a patient's best interests take precedence over any profit motive. ${ }^{4}$ With this in mind, recommendations for use of an LLLT device to treat hair loss begins with recommending only those devices independently evaluated and cleared by a regulatory body for safety (in the United States, this is $510(\mathrm{k})$ clearance), and that have provided acceptable medical evidence of efficacy beyond anecdotal response. Preferably, a randomized controlled trial. However, as this review will address, even data from randomized controlled trials need to be evaluated with a critical understanding of both recognized hair growth promoter assessment techniques as well as the principles and limitations governing LLLT and biostimulation. Many hair research doctors are well versed in the former, and laser therapy specialists the latter-a critical review of the available LLLT trials for hair growth suggests a need to better integrate the two areas of expertise.

In 2013-2014, there were four newly published "randomized controlled trials" studying various LLLT devices for treating hair loss. ${ }^{5-8}$ Three studies involved helmets with multiple diode lasers, some of which included LED lights. The fourth and largest study assessed response to several models of a laser comb, each with increasing numbers of laser diodes-7, 9, and $12 \mathrm{di}$ odes, respectively. All of the four published studies considered increases in hair counts in a small study area as an endpoint. All studies used a recognized methodology for assessing hair counts, using a single tattoo on the specified scalp study area, where hair was trimmed prior to obtaining "before" and "after" phototrichograms. Global photo assessment with "before" and "after" comparisons were done in the Laser Comb and Korean helmet study, but not in the two U.S. "Top Hat" helmet trials. ${ }^{5-8}$ None of these trials used stabilization of hair loss as an endpoint, and could not have done so because of the short trial duration (26 weeks or less). Furthermore, none of the studies described how they determined the "dose" of therapy (duration and frequency of treatment) and, therefore, did not address the possibility of a biphasic dose response risk where too short or too long an exposure would have either no impact or adverse impact on hair growth. In addition, none of the studies addressed which of the multiple diode lasers or LEDs were positioned to effectively biostimulate the single area trimmed for the hair counts. In contrast to systemic treatments (finasteride) or topical applications to the skin (minoxidil) where hair trimming made hair counts easier to perform, researchers for the LLLT studies did not address how trimmed hair vs. the presence of hair could be confounding variables for the application of light therapy to scalp. Realizing the effect of these variables suggests that study designers should have developed an alternate method or used standardized hair pattern and hair color characteristics for evaluating the same treatment application. Each study reached varying degrees of a positive conclusion regarding the effectiveness of their LLLT device to treat hair loss. However, closer scrutiny of data, methodology, short study duration, as well as an awareness of the scientific principles of biostimulation, biphasic dose response, light properties, and melanin as a chromophore in skin and hair must be considered when assessing the validity and utility of information obtained from these trials.

\section{Performing Accurate Hair Counts \& Comparing Phototrichograms}

When evaluating phototrichogram data intended to compare hair counts, investigators must be experienced in photo preparation of the study area as well as how to photograph for uniform "before" and "after" data collection accuracy and ease of comparison. An awareness of how to interpret or evaluate this methodology is important for readers to understand, in order to evaluate the accuracy of study conclusions. Hair counts are tedious and difficult and require adherence to specific comparative techniques. Most studies utilize a single tattoo in the midpoint of a study area, usually $1-2 \mathrm{~cm}^{2}$ where a phototrichogram (magnified photo of follicular units) is taken. (In the U.S. helmet studies, this area was reportedly $2.5 \mathrm{~cm}^{2}$ ). When a single dot tattoo is used, rather than 2 dots, it is more difficult to avoid frame of reference errors because rotating a camera only $1 \mathrm{~mm}$ north, south, east or west of the dot can potentially change the number of follicular units/hairs included in the assessment area. Hair length is a critical factor for accuracy in hair counts, too. Too short or completely shaved hairs will not be visible for counting. However, researchers do use the shaving method as a means to determine anagen from telogen hairs - by shaving and then photographing 3-5 days later, you can discern which hairs are actually growing (anagen) from those in the telogen phase. The photos taken at 3-5 days would then constitute the baseline phototrichogram for counting hairs before and after treatment. No apparent efforts were made to assess anagen from telogen hairs in these studies, and this might influence study results. Trimmed hairs left too long $(>1-2 \mathrm{~mm})$ allow hairs to overlap, obscuring those beneath or adjacent. These factors are especially important for automated counting methods-none of which have yet proven accurate without some form of manual assistance. Methods to standardize hair length to $1 \mathrm{~mm}$ will optimize 
counting ease and accuracy, and when not used will call accuracy into question. ${ }^{9,10}$ Furthermore, comparing phototrichograms visually where hair is longer in an after photo is misleading, as longer length creates the illusion of greater coverage. Phototrichogram images can be enlarged on a computer screen, follicular units marked and counted, with sentinel groupings to ensure perspective is the same and area perimeters are identical.

Because opportunity exists for error using these methodologies, particularly if performed or interpreted by inexperienced photographers, it is imperative that published hair count data be accompanied by sufficient numbers of "before and after" phototrichograms and global photos to establish the credibility of reported results. Notably, none of these trials had multiple examples included in their publication to support their data or positive conclusions. The most accurate and credible response example was provided in the Laser Comb study where global photos of a female patient were substantiated with accurately illustrated before and after photo trichograms - marking sentinel groupings and new hairs. However, as was pointed out by an astute researcher (verbal communication), it is remarkable that in a study where more than 100 patients were treated, only one notable example was published. As previously mentioned, there were no phototrichograms or global photos included in the Korean helmet study, and no global photos in the two U.S. "Top Hat" helmet studies. Of the phototrichograms provided in the U.S. helmet studies, when hairs were counted, results did not match the labeled numbers of purported increased hairs. The latter point illustrates the importance of including these photos, so reviewers may confirm for themselves and avoid drawing clinical conclusions from potentially inaccurate hair count reports.

\section{Comparison LLLT Device Data with Established Hair loss Treatment and Each Other}

Credibility of hair count data accuracy, when not readily corroborated by visual assessment, can also be suggested by comparing reported hair counts to results from other credible studies of recognized hair growth promoters. Dramatic hair growth claims should be an indication for closer scrutiny. Table 1 compares device characteristics including wavelength, number of laser diodes, and LED lights, as well as dosing regimens or energy (E) applied in each of the randomized, controlled trials. In order to compare device results, similar data points such as percentage hair count increase or absolute hair counts were used and can be found in Table 2. Absolute hair counts from raw data were available for the smaller helmet studies, but not for the largest study with the Laser Comb. In order to compare the helmet studies with the Laser Comb, the data point of percentage of patients with an increase $>20$ hairs $/ \mathrm{cm}^{2}$ was used, as this appeared to be the parameter demarcating response to an LLLT laser comb above growth generated by the sham (placebo) device. Table 2 summarizes and compares the percentage of patients in each device study group (LLLT vs. sham) that achieved hair growth results either $>20$ hairs $/ \mathrm{cm}^{2}$ or $>15 \%$ increases in hair counts during the study period. These comparisons made it possible to understand the substantial variations in reported increased hair counts between these devices, and to compare them to increases in hair counts among patients treated with the FDA-approved drug, finasteride. Particular scrutiny was engendered by extraordinary growth claims from the U.S. helmet studies.

\begin{tabular}{|c|c|c|c|c|c|}
\hline Device & $\underset{M / F}{N}$ & $\begin{array}{l}\text { Wavelength (nm) } \\
\text { Laser Diode (LD) } \\
\text { LED }\end{array}$ & $\begin{array}{l}\text { Power } \\
(\mathrm{mW}) \\
\text { Area (A) }\end{array}$ & $\begin{array}{l}\text { Dur/Freq } \\
\text { PD for one } \\
\text { LD or LED } \\
\text { E (J)= Watts (P) X s }\end{array}$ & $\begin{array}{l}\text { Study } \\
\text { Lengt } \\
\mathrm{h} \\
\text { (wks) }\end{array}$ \\
\hline $\begin{array}{l}\text { Laser } \\
\text { Comb } \\
\text { (moving) } \\
\text { FDA-510K }\end{array}$ & $\begin{array}{l}128 \mathrm{M} \\
141 \mathrm{~F}\end{array}$ & $\begin{array}{r}7,9 \text { beam: } 655 \mathrm{~nm} \\
12 \text { beam: } 6-655 \mathrm{~nm} \\
6-635 \mathrm{~nm}\end{array}$ & $\begin{array}{l}P=5 \mathrm{~mW} \\
(.005 \mathrm{~W}) \\
\text { Beam } \\
A=?\end{array}$ & $\begin{array}{l}15,11 \mathrm{~min} \\
8 \mathrm{~min} \\
\mathrm{X} 3 / \mathrm{wk}- \\
\text { moving } 1 \mathrm{~cm} / \\
4 \mathrm{~s} \\
\mathrm{E}=.02 \mathrm{~J}\end{array}$ & 26 \\
\hline $\begin{array}{l}\text { LLLT/LED } \\
\text { Helmet } 1 \\
\text { stationary } \\
\text { KFDA }\end{array}$ & $\begin{array}{l}26 \mathrm{M} \\
14 \mathrm{~F}\end{array}$ & $\begin{array}{l}27 \text { LD @ } 650 \mathrm{~nm} \\
24 \text { LED@630 nm } \\
18 \text { LED@660 nm } \\
\text { ( } 69 \text { beams) }\end{array}$ & $\begin{array}{l}P=4 \mathrm{~mW} \\
P=3.5 \mathrm{~mW} \\
P=2.5 \mathrm{~mW} \\
A=?\end{array}$ & $\begin{array}{l}18 \mathrm{~min} / \mathrm{d} \\
E=4.32 \mathrm{~J} \\
E=3.78 \mathrm{~J} \\
E=2.7 \mathrm{~J}\end{array}$ & 24 \\
\hline $\begin{array}{l}\text { LLLT/LED } \\
\text { Helmet } 2 \\
\text { stationary } \\
\text { FDA SLIoK }\end{array}$ & $\begin{array}{l}44 \mathrm{M} \\
47 \mathrm{~F}\end{array}$ & $\begin{array}{l}21 \mathrm{LD} \quad 655 \mathrm{~nm} \\
30 \text { LED } \quad 655 \mathrm{~nm} \\
\text { (51 beams) }\end{array}$ & $\begin{array}{l}P=5 \mathrm{~mW} \\
\text { Beam } \\
A=?\end{array}$ & $\begin{array}{l}25 \mathrm{~min}, \text { qod } \\
E=7.5 \mathrm{~J}\end{array}$ & 16 \\
\hline
\end{tabular}

Table 1. Comparing Light Parameters and Dosing Schedules for LLLT Devices in This Report

\begin{tabular}{|c|c|c|c|c|}
\hline $\begin{array}{l}\text { Device } \\
\text { N=LLLT/Sham }\end{array}$ & $\begin{array}{l}\% \text { Pts } \\
>20 \text { hair/ } \\
\text { cm2 } \\
\text { LLLT }\end{array}$ & $\begin{array}{l}\% \text { Pts } \\
>20 \text { hair } / \mathrm{cm} 2 \\
\text { Sham }\end{array}$ & $\begin{array}{l}\% \text { Pts } \\
>15 \% \text { incr } \\
\text { LLLT }\end{array}$ & $\begin{array}{l}\% \text { Pts } \\
>15 \% \text { incr } \\
\text { Sham }\end{array}$ \\
\hline $\begin{array}{l}\text { L Comb-M } \\
7,9,12 \text { beam } \\
67 / 36\end{array}$ & $38-68 \%$ & $0-14 \%$ & $\begin{array}{l}\text { N/A } \\
\text { No raw data }\end{array}$ & $\mathrm{N} / \mathrm{A}$ \\
\hline $\begin{array}{l}\text { L Comb-F } \\
9,12 \text { beam } \\
82 / 40\end{array}$ & $40-41 \%$ & $5.5 \%$ & N/A & N/A \\
\hline $\begin{array}{l}\text { Helmet } 1 \mathrm{M} / \mathrm{F} \\
\text { 15/14 }\end{array}$ & $26.6 \%$ & $7.1 \%$ & $\begin{array}{l}33 \% \\
\text { Ave } 29 \%\end{array}$ & $\begin{array}{l}14.3 \% \\
\text { Ave } 25 \%\end{array}$ \\
\hline $\begin{array}{l}\text { Helmet 2-M } \\
22 / 19\end{array}$ & $72.7 \%$ & $21.0 \%$ & $\begin{array}{l}100 \% \\
\text { Ave } 67.2 \%\end{array}$ & $\begin{array}{l}37 \% \\
\text { Ave } 75.7 \%\end{array}$ \\
\hline $\begin{array}{l}\text { Helmet 2-F } \\
24 / 28\end{array}$ & $79.1 \%$ & $3.6 \%$ & $\begin{array}{l}95.8 \% \\
\text { (Ave 50\% } \\
\text { increase) }\end{array}$ & $\begin{array}{l}30 \% \\
\text { (Ave } 24 \% \text { increase) }\end{array}$ \\
\hline
\end{tabular}

Table 2. Hair Count Comparisons: Laser Comb, Korean Helmet, U.S. “Top Hat" Helmet

In performing data reanalysis, first the LLLT device data was compared to an average 15\% increase in hair counts achieved with the FDA-approved finasteride. Previous published reports of response to finasteride revealed an average increase in hair counts after a year of therapy was $14 \%$, and rose to $16 \%$ at 2 years (mean increase 15\%). ${ }^{11}$ The three published helmet studies were sufficiently small to include raw data for re-analysis to make this comparison. In the Korean helmet group, 33\% of patients achieved a reported $>15 \%$ increase in hair counts-similar to increases seen with finasteride treatment-interestingly, so did $14.3 \%$ of placebo patients. When assessed by absolute hair counts, $26.6 \%$ of patients had increased $>20$ hairs $/ \mathrm{cm}^{2}$, compared to $7.1 \%$ in the placebo group. Coincidentally, the same percentage of treated patients who experienced increases $>20$ hairs $/ \mathrm{cm}^{2}$ were noted by investigators and patients to have a visible global improvement, but this comparison (hair counts to visible global improvement) were not done. What this finding also illuminates is that nearly $70 \%$ of treated patients did not experience a significant clinical response. 
Part 3: LLLT from page 103

There were two separate published studies sponsored by the U.S. helmet manufacturer referred to as the "Top Hat" device. One study included only males and another, only females. (Notably one of the authors of the study, R.J. Lanzafame, has received consulting fees and fees for manuscript preparation, and has ownership interest in Apira Science- the device manufacturer). Remarkably, raw data in the male study revealed a surprising number of patients in both the placebo and the treatment group who reported to have $100 \%$ increases in hair counts. However, there were no published global photos. Phototrichograms, which were published, did not support hair growth claims. A visual analysis of the published phototrichograms suggested errors in hair counting methods and photography. In both studies, published phototrichograms revealed non-uniformity in hair length and camera perspective. More importantly, as noted, reported increases in hair counts, as labeled in the article, were substantially inaccurate by my manual count-raising doubts about the accuracy of all data reported. In the male study, the percentage of patients with a $>15 \%$ increase was $100 \%$, and nearly $96 \%$ for the female study. But not only did these reports indicate a growth response comparable to those seen with finasteride-achieved after only 4 months of therapy-they far exceeded them. In the male study, average increased hair counts were $65 \%$ (vs. $15 \%$ in finasteride-treated patients); for women, this number was a whopping $50 \%$ increase. Both of the published studies regarding this device were small (males: 22 treated, 19 sham; females: 24 treated, 28 sham), and as noted, the study duration was only 16 weeks.

In the fourth study to be evaluated, the Laser Comb study had the largest number of patients $(\mathrm{n}=225)$ and did not present raw data. Therefore, it was not possible to determine what percentage of patients achieved a $>15 \%$ increase in hair counts, which, as noted, is comparable to finasteride response in men. However, in reviewing the bar graphs for treated patients with increases in hair counts $>20$ hairs per square centimeter, the Laser Comb achieved this for 38$68 \%$ of men, depending on the number of laser diodes; for women, this was $40-41 \%$. These numbers also reveal that the number of non or minimal responders was significant; for women, $60 \%$ of patients did not achieve a growth response $>20$ hairs $/ \mathrm{cm}^{2}$, and for men, this range was $32-40 \%$. Again, there was insufficient phototrichogram evidence or global photos to support efficacy claims.

\section{Other Limitations and Caveats}

In addition to questions raised by the paucity or absence of phototrichogram evidence-as well as questions raised when LLLT studies boast hair count increases far beyond those achieved by proven medical therapies-other questions remain unanswered, too. In the Laser Comb study, nearly $50 \%$ of placebo patients reported "minimal to improved" global appearance, and this response report was increased by approximately $10 \%$ among patients who were actually treated. Even if hair counts in the study area are assumed accurate, is there another reason this effect cannot be observed? Furthermore, since none of the studies provided longterm follow-up, what evidence exists to suggest gains could be sustained? In fact, considering the known biphasic response nature of biostimulation, it is expected an LLLT dose exists where excessive duration of stimulation may actually cause follicle damage. This question is pertinent to the fact that none of the studies addressed dosing, or discussed how duration and frequency of treatment was determined in order to avoid the biphasic response. This question is further pertinent to the development of devices that boast higher energy levels, without evidence that higher energy will improve results, or that this may actually have the opposite effect on hair growth. There are a number of published studies in animal models that describe the phenomenon and how dosimetry $\left(\mathrm{J} / \mathrm{cm}^{2}\right)$ and time are critical to achieving a biostimulatory effect—or to producing no effect at all. For example, differences in irradiance matter. In a hamster model of mucositis treatment, using a $660 \mathrm{~nm}$ laser in the first group at $55 \mathrm{~mW} / \mathrm{cm}^{2} \times 16 \mathrm{sec}$ and in the second group at $155 \mathrm{~mW} / \mathrm{cm}^{2} \times 6 \mathrm{sec}$-both with comparable $\left(\mathrm{J} / \mathrm{cm}^{2}\right)$ —only group 1 had a positive response. ${ }^{12}$ The extent to which this phenomenon is seen in human patients is not yet known, but is believed to be the reason for some ineffective LLLT trials.

Another looming, unanswered question was raised in Part 1 of this series, and observes the basic principle of biostimulation that provides that a particular wavelength creates a cellular response when absorbed by a cellular target. Biostimulation can only occur if light reaches its target in the skin at the proper dose. Melanin in hair and skin is a chromophore absorbing laser light - preventing it from penetrating skin to reach cellular targets below. Depending on the angulation of a light beam, it can also be completely reflected, so, for example, laser helmet beams on the side of a helmet if beamed tangentially against hair on the top of the scalp will be reflected and cannot reach cellular targets there. As reported in Part 1 of this series, computer models estimate melanin in hair can reduce light transmission to the skin by close to $40 \%$ at an assumed length of only a few mm. ${ }^{13}$ It should be expected this will be further reduced by long or layered hair, especially black hair containing the most absorptive pigment, eumelanin. A similar computer model verified the effect of skin color in reducing transmissible light for photodynamic therapy_indicating that medium to dark skin significantly impedes light transmission. ${ }^{14}$ Dosing schedules that are the same despite differences in hair length, hair color, and skin color, cannot be assumed to render equal biostimulation to cellular targets. Unless patients have minimal hair or a shaved head for treatment, it is not clear how studies or LLLT devices will overcome this obstacle. Devices that part the hair and are moveable across the scalp are likely to reach more cellular targets. However, even these devices will have their limitations when dosing regimens ignore the known relevance of hair and skin color.

A similar limitation in the ability of LLLT to reach all cellular targets on the affected scalp must be considered for stationary devices - where light beams can only affect cells in their path. Light transmission is affected by distance and angulation, in addition to chromophores. LED lights, as mentioned in Part 1, cover a broader area as they are less coherent and collimateddistance from the skin reduces their power, while proximity to skin reduces their area of coverage. At no time did researchers indicate which of the light beams was being applied to a given study area - neither were explanations offered as to why multiple light beams were part of a device, and how the study model was designed to assess each of them. In fact, study methodology made it impossible to know whether various wavelengths used had equal utility, or contribution to results. Presumably, if each laser diode or LED were comparable in biostimulating properties, there would be no need for variation. Regardless, these studies did not provide information to elucidate these questions. 


\section{Conclusion}

What can we advise patients who ask about LLLT for treating their hair loss? To summarize, evidence supporting efficacy of current LLLT devices is fraught with limitations due to erroneous or improper use of existing hair growth promoting assessment methodology and with study designs that don't consider known variables affecting light transmission for biostimulation. Therefore, no accurate conclusions can yet be reached, and recommendations should include the caveats listed here. Hair counting methods require hair to be cut short in the study area, and this disturbs the ability to evaluate the effect of LLLT in the case of patients who have pre-existing, longer hair everywhere but the study area. While LLLT may work for some patients (short hair, decreased pigment: blonde, fair skin), credible evidence (phototrichograms, global photos) to support effectiveness for MOST patients who use these devices is lacking. Important questions about dosing for LLLT therapy remain unanswered.

Only those devices $510(\mathrm{k})$ cleared are legal for sale in the United States, and it behooves patients everywhere to make sure any medical device they purchase has been evaluated for safety—mislabeled lasers are a safety hazard.

\section{Future Research Recommendations}

In reviewing copious literature establishing the science of biostimulation, it seems if we can identify the optimal wavelength and adjustable dosing regimens, and create the device that can bypass hair and skin to uniformly reach cellular targets, we should be able to achieve a hair growth benefit. Even so, existing research using LLLT for biostimulation has not determined if results from these devices can be sustained.

Nevertheless, there are patients unable to tolerate existing therapies such as finasteride or topical minoxidil. An alternate effective therapy could be very useful. The in vitro data from cultured cells documented a variety of wavelength ranges that would optimize stimulation of DNA synthesis, which, ostensibly, promotes growth factors to grow hair. As previously noted in this series, only one device currently cleared for use (a helmet) includes a wavelength promoting optimal DNA synthesis in cell cultures. This device has not been used to conduct a controlled study. The optimal study population for standardized LLLT device evaluation are men with moderate thinning, very short/shaved blonde hair, and fair skin —so identical dosing regimens may be used and the same light dose would be expected to pass through hair and skin to reach cellular targets. Who will conduct this study? As long as patients continue to purchase existing LLLT devices, and doctors continue to sell or recommend them, it is unlikely businesses will be motivated to test alternate wavelengths or dosing regimens. Inherent limitations in study design and clinical application will apply as long as dosing schedules fail to consider melanin or other chromophores in hair and skin - and as long as stationary application of light beams occur that cannot hope to stimulate cellular targets outside their beams.

\section{References}

1. Akobeng, A.K. Understanding randomised controlled trials. Arch Dis Child. 2005; 90:840-844.

2. http://www.accessdata.fda.gov/drugsatfda_docs/nda/2006/ 021812s000_MedR.pdf

3. www.fda.gov, 2014

4. http://virtualmentor.ama-assn.org/2009/05/ccas2-0905.html

5. Jiminez, J., et al. Efficacy and safety of a LLLT device in the treatment of male and female pattern hair loss: a multicenter, randomized, sham device-controlled, double-blind study. Am J Clin Derm. 2014; 15:115-127.

6. Kim, H., et al. LLLT for AGA: a 24-week, randomized, double-blind, sham device controlled multi-center trial. Derm Surg. 2013(Aug).

7. Lanzafame, R., et al. The growth of human scalp hair mediated by visible red light laser and LED sources in males. Lasers in Surgery \& Med. 2013; 45:487-495.

8. Lanzafame, R., et al. The growth of human scalp hair in females using visible red light laser and LED sources. Lasers in Surgery \& Med. 2014; 46:601-607.

9. Van Neste, D. Variants of phototrichogram methods. Bioengineering of the Skin: Skin Imaging \& Analysis. 2006(Sep); 282.

10. Dhurat, R. Phototrichograms, Indian J Dermatol Venereol Leprol. 2006; 72(3).

11. Shapiro, J., and K. Kaufman. Use of finasteride in the treatment of men with AGA. J Invest Derm Symp Proceedings. 2003; 8:20-23.

12. Huang, Y.Y., et al. Biphasic dose response in low level light therapy - an update. Dose Response. 2011; 9:602-618.

13. Mustafa, F.H., et al. Influence of hair color on photodynamic dose activation in PDT for scalp disease. IFMBE proceedings. 2011; vol 35.

14. Farhad, H., et al. Red diode laser in the treatment of epidermal diseases in PDT. World Academy of Science, Engineering and Technology. 2010; 46:779-783.

\section{Stop by our booth at the Chicago meeting! \\ Our New Pouches- Easy to Pack for Patient Travel}

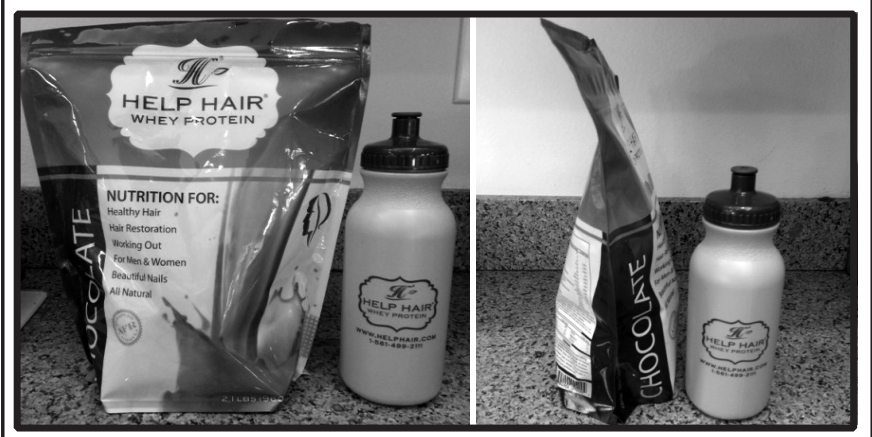

RSVP:

Chicago Deep Dish Pizza Party info@HelpHair.com 1-561-499-2111 